\title{
Three dimensional configuration of earthward fast plasma flow in the near-Earth plasma sheet
}

\author{
Koji Kondoh, Masayuki Ugai, and Tohru Shimizu \\ Research Center for Space and Cosmic Evolution, Ehime University, Japan \\ (Received November 2, 2007; Accepted August 16, 2008; Online published May 29, 2009)
}

\begin{abstract}
The earthward short-term (1-min. order) fast flow event (Flow Burst) and the long-term (10-min. order) fast flow event (Bursty Bulk Flow) observed in the near-Earth plasma sheet are examined using three dimensional MHD simulations on the basis of spontaneous fast reconnection model. It is well known that these fast flow events are closely related to the magnetic substorms. On the other hand, it is considered that these fast flow events are caused by the magnetic reconnection in the near-Earth magnetotail. The time profiles of plasma quantities in these events observed by in-situ satellites are quite different in each event. Above Flow Burst and Bursty Bulk Flow events are often examined separately due to the large difference in time scale. In this paper, these differences are interpreted by the three-dimensional position of satellite relative to the X-line and the reconnection jet channel, and the simulation results are directly compared with the results of in-situ satellite observations using the virtual satellites located in simulation domain.
\end{abstract}

Key words: Earthward flow, magnetic reconnection, near-Earth plasma sheet, three-dimensional MHD simulation.

\section{Introduction}

Earthward fast flows in the near-Earth plasma sheet have been observed in association with geomagnetic substorms (Angelopoulos et al., 1992). It is considered that these fast flows are fast reconnection outflows. In space plasmas of extremely large magnetic Reynolds number, magnetic reconnection can convert magnetic energy to plasma energies most effectively by slow shocks. A possible fast reconnection configuration, involving standing slow shocks, was first proposed by Petschek (1964). In this respect, we have proposed the spontaneous fast reconnection model. This model predicts that the fast reconnection mechanism spontaneously develops from inside the system by the selfconsistent interaction between plasma microscopic processes and macroscopic reconnection flows (Ugai, 1984, 1986). The previous MHD simulations have demonstrated that the spontaneous fast reconnection model works quite effectively even in three dimensional situations (Ugai and Kondoh, 2001; Ugai et al., 2004, 2005). Once the fast reconnection builds up, an Alfvénic fast flow is caused, and if the fast flow is obstructed, the reconnected field lines are piled up, and a magnetic loop is formed. A magnetic loop in our simulation is allowed to be formed because of a wall boundary assumed at one edge of a current sheet system (Ugai et al., 2003). Sergeev et al. (2000) showed a narrow transient flow jet using five spacecraft measurements. They showed that the $\sim 3 R_{\mathrm{e}}$ wide fast plasma jet propagates from $\gtrsim 40 R_{\mathrm{e}}$ in the midtail, and is able to reach the inner magnetosphere at $6.6 R_{\mathrm{e}}$ in about 10 minutes. This property

Copyright (c) The Society of Geomagnetism and Earth, Planetary and Space Sciences (SGEPSS); The Seismological Society of Japan; The Volcanological Society of Japan; The Geodetic Society of Japan; The Japanese Society for Planetary Sciences; TERRAPUB. consists with the previous results of MHD simulations on the basis of spontaneous fast reconnection model (Ugai et al., 2003).

Earthward fast flow events observed in the near-Earth plasma sheet have the different profiles in each event. For example, Figs. 1(a) and (b) are the different two events observed by Geotail satellite. These figures show the time profiles of the plasma bulk velocity component $V_{x}$ (top panel), the magnetic field components $B_{x}$ (dotted line), $B_{z}$ (solid line) (middle panel), and the ion density $N$ (bottom panel). These satellites observed at $X=-29.67 R_{\mathrm{E}}, Y=$ $7.50 R_{\mathrm{E}}, Z=-1.26 R_{\mathrm{E}}$ (a), $X=-28.31 R_{\mathrm{E}}, Y=2.65 R_{\mathrm{E}}$, $Z=-2.03 R_{\mathrm{E}}$ (b) in GSM coordinate, respectively. In GSM coordinate system, these events were observed at very near positions. The maximum velocity of $V_{x}$ is almost same, $600 \mathrm{~km} / \mathrm{s}$. As you can see, however, these two events are quite different. The duration of the enhancement of flow velocity in Fig. 1(a) ( $\sim 7 \mathrm{~min}$.) is remarkably longer than that in Fig. 1(b) ( $\sim 1$ min.). The variation of the ion density in the course of fast flow is large in Fig. 1(a), while that is little in Fig. 1(b). In this paper, we examine the earthward fast flow using three-dimensional MHD simulations on the basis of spontaneous fast reconnection model, and what causes these differences.

\section{Simulation Model}

The earthward fast flow is closely related to the formation of magnetic loop (or magnetic dipolarization), so that the present simulation model is similar to the one of the 3D magnetic loop dynamics (Kondoh and Ugai, 2007).

These previous magnetic loop simulations using the wall boundary showed that the fast magnetic reconnection produces fast reconnection jet, and it flows in plasma sheet and 


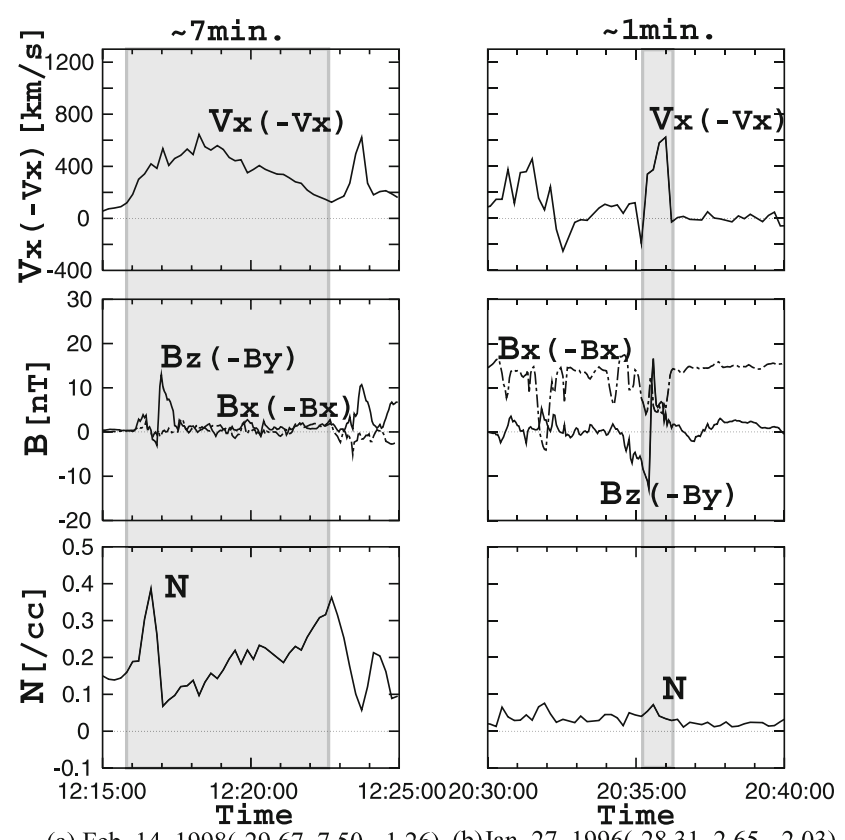

(a) Feb. 14, 1998(-29.67, 7.50, -1.26) (b)Jan. 27, 1996(-28.31, 2.65, -2.03)

Fig. 1. Geotail observation data showing the plasma bulk velocity component $\left(V_{x}\right)$, magnetic field components $\left(B_{x}, B_{z}\right)$ and ion density $(N)$ (12:15-12:25 UT, 14 February 1998 (a), 20:30-20:40 UT, 27 January 1996 (b)). The variables in parenthesis indicate the corresponding ones in our coordinate system.

is suddenly braked at the boundary between the dipolar and tail-like magnetic field because of the counterward pressure force, and the reconnection jet is decelerated. This situation may be consistent with the earthward fast flow, and these results are in good agreement with the situation inferred from the observation results of earthward fast flow (Shiokawa $e t$ al., 1997).

\subsection{Simulation modeling}

As an initial configuration, the one-dimensional antiparallel magnetic field $\mathbf{B}=\left[B_{x}(y), 0,0\right]$ is assumed as: $B_{x}(y)=\sin (\pi y / 2)$ for $0<y<1 ; B_{x}(y)=1$ for $1<$ $y<Y_{1} ; B_{x}(y)=\cos \left[\left(y-Y_{1}\right) \pi / 1.2\right]$ for $Y_{1}<y<Y_{m}(=$ $\left.Y_{1}+0.6\right) ; B_{x}(y)=0$ for $Y_{m}<y$; also, $B_{x}(y)=-B_{x}(-y)$ for $y<0$. The plasma pressure $P(y)$ initially satisfies the pressure-balance condition, $P+B_{x}^{2}=1+\beta_{0}$, where $\beta_{0}$ is the ratio of plasma pressure to the magnetic pressure in the ambient magnetic field region $1<y<Y_{1}$, so that $P(y=0)=1+\beta_{0}$ initially (in the present study, $\beta_{0}=0.15$ is taken); Initially, fluid velocity $\mathbf{u}=(0,0,0)$ and constant temperature $T=P / \rho=1+\beta_{0}$ is assumed, so that the plasma density $\rho$ initially satisfies $\rho(y)=P(y) /\left(1+\beta_{0}\right)$. The normalization of quantities, based on the initial quantities, is self-evident; Distances are normalized by the halfwidth of the current sheet $d_{0}, \mathbf{B}$ by the field strength in the magnetic field region $B_{x 0}, P$ by $B_{x 0}^{2} /\left(2 \mu_{0}\right)$, and $\rho$ by $\rho_{i}=\rho(y=0)$; also, u by $V_{A x 0}\left(=B_{x 0} / \sqrt{\mu_{0} \rho_{i}}\right)$, time $t$ by $d_{0} / V_{A x 0}$, current density $\mathbf{J}$ by $J_{0}=B_{x 0} /\left(\mu_{0} d_{0}\right)$, and so forth.

Here, the conventional symmetry boundary conditions are assumed on the $(x, y),(y, z)$, and $(z, x)$ planes. Hence, the computational region can be restricted to the first quadrant only and taken to be a rectangular box, $0<x<L_{x}$,
$0<y<L_{y}$, and $0<z<L_{z}$; also, for simplicity, the conventional symmetry boundary condition is assumed on the outer boundary plane $x=L_{x}$, and on the other boundary planes ( $y=L_{y}$ and $z=L_{z}$ ) the free boundary conditions are assumed.

As in the 2D model, a current-driven anomalous resistivity model is assumed in the form,

$$
\begin{aligned}
\eta(\mathbf{r}, t) & =k_{\mathrm{R}}\left[V_{\mathrm{d}}(\mathbf{r}, t)-V_{\mathrm{C}}\right] & & \text { for } V_{\mathrm{d}}>V_{\mathrm{C}}, \\
& =0 & & \text { for } V_{\mathrm{d}}<V_{\mathrm{C}}
\end{aligned}
$$

where $V_{\mathrm{d}}(\mathbf{r}, t)=|\mathbf{J}(\mathbf{r}, t) / \rho(\mathbf{r}, t)|$ is the relative electronion drift velocity, and $V_{\mathrm{C}}$ may be a threshold for micro instabilities. Here, $k_{\mathrm{R}}=0.003$ and $V_{\mathrm{C}}=12$ are taken.

In order to disturb the initial static configuration, a localized resistivity model is assumed around the point $\left(L_{x}, 0,0\right)$ in the 3D form,

$$
\eta(\mathbf{r})=\eta_{0} \exp \left[-\left(\left(x-L_{x}\right) / k_{x}\right)^{2}-\left(|y| / k_{y}\right)^{3}-\left(|z| / k_{z}\right)^{3}\right]
$$

Here, we take $k_{x}=k_{y}=0.8$ and $\eta_{0}=0.02$ in the manner similar to the previous $2 \mathrm{D}$ simulations; also, $k_{z}$ provides the $3 \mathrm{D}$ effects, we take $k_{z}=5$ in the present study. The disturbance (2) is imposed only in the initial time range $0<t<4$, and the anomalous resistivity model (1) is assumed for $t \geq 4$. Hence, the fast reconnection mechanism may be triggered at $x=L_{x}$ in this model.

It should be noted that sufficiently small mesh sizes are required for precise computations of the spontaneous fast reconnection evolution, so that we assume $\Delta x=$ $0.04, \Delta y=0.015$, and $\Delta z=0.1$. Also, we take the magnetic field region size $Y_{1}=4$, and the whole computational region size is assumed to be $L_{x}=10, L_{y}=9.6$ and $L_{z}=9.8$. Here, we should notice that positive $x, y, z$ directions in this paper correspond to tailward, south-ward, and east-ward in usual GSM co-ordinate system, respectively.

\section{Results}

In this simulation model, initiated by the disturbance (2) given at $x=L_{x}$ in the finite extent $|z|<k_{z}=5$, the current sheet thinning occurs near the $x=L_{x}$, giving rise to distinct enhancement of the current density. Once the drift velocity $V_{\mathrm{d}}$ exceeds the given threshold (1), fast reconnection drastically grows as nonlinear instability because of the positive feedback between the anomalous resistivity and the reconnection flow. Ahead of the Alfvénic reconnection jet flowing to negative $x$-direction, a large-scale 3D plasmoid is formed and propagates, and the plasmoid collides with the $x=0$ wall boundary, giving rise to a 3D magnetic loop.

\subsection{Virtual observations}

In order to directly compare the simulations with the satellite observations, let us consider such a virtual satellite that is located at a spatial point $(x, y, z)$ in the plasma sheet. For simplicity, we assume that the satellite does not move in spite of the progress of time. Then, the virtual satellite can readily detect the temporal changes of plasma quantities at its location on the basis of the simulation results. In what follows, we examine what the virtual satellite observes as the fast reconnection mechanism builds up and proceeds. 

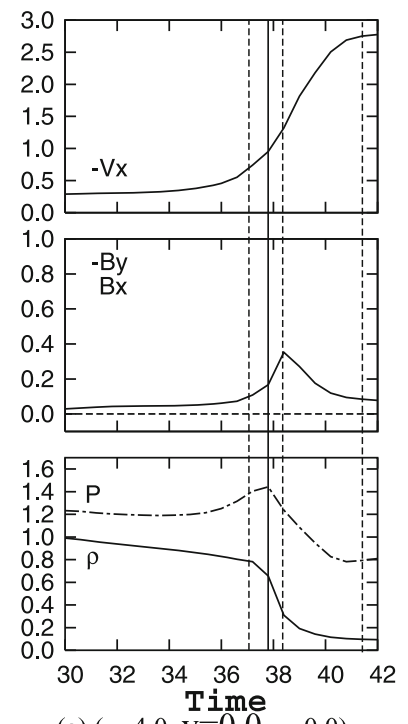

(a) $(\mathrm{x}=4.0, \mathrm{y}=0.0, \mathrm{z}=0.0)$
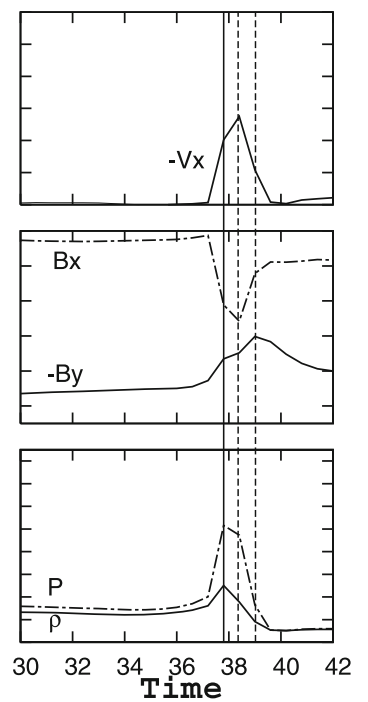

(b) $(\mathrm{x}=4.0, \mathrm{y}=0.8, \mathrm{z}=0.0)$

Fig. 2. Time variations of $-V_{x}, B_{x},-B_{y}, \rho$, and $P$ for the interval of $t=30$ to 42 at $X=4.0, Z=0.0, Y=0.0$ (a) and 0.8 (b).

At first, two virtual satellites are located at the positions $(4.0,0.0,0.0)$ and $(4.0,0.8,0.0)$ in order to examine the profiles at different $y$-positions. These positions correspond to them in the central plasma sheet (hereafter, call CPS) and near the plasma sheet boundary layer (hereafter, call PSBL), respectively. Figure 2 shows the temporal variations of the plasma flow velocity (top panel), magnetic field components (middle panel), plasma pressure and plasma density (bottom panel) detected by the virtual satellites located at $(4.0,0.0,0.0)$ (a) and $(4.0,0.8,0.0)$ (b). In the CPS (a), the earthward flow velocity $V_{x}$ reaches Alfvén velocity. The local magnetic field dipolarizes a little. $P$ and $\rho$ largely decrease in the course of the fast flow, and the temperature becomes high there. On the other hand, near the PSBL (b), the enhancement of $V_{x}$ is small and short, $B_{x}$ largely decrease in the course of fast flow. The clear dipolarization is also detected. The durations of the variations in $P$ and $\rho$ are nearly same and short. The temperature in the course of the fast flow is high as well as that in the CPS. The peak times of the pressure enhancement $T_{P}$ indicated by the vertical solid lines are almost same $t \sim 37.7$. The vertical dotted lines indicate the peak times of the other quantities. The earthward flow speed $\left|V_{x}\right|$ at $T_{P}$ are almost same $\sim 1.0$ at both positions. The earthward flow speed in the CPS gradually increase from $t \sim 30$, whereas it near the PSBL starts to increase just before $T_{P}$. The anti-correlation between the earthward flow speed $\left|V_{x}\right|$ and earthward component of magnetic field $B_{x}$ is clear in Fig. 2(b). This rapid increase (decrease) and decrease (increase) of $\left|V_{x}\right|\left(B_{x}\right)$ near the PSBL means that the satellite suddenly goes in the fast flow channel and suddenly goes out there.

Secondly, the other two virtual satellites are located at $(4.0,0.4,0.0)$ and $(4.0,0.4,1.2)$ in order to examine the profiles at different $z$-positions. These positions correspond to the those in the channel of Alfvénic fast flow (hereafter, call fast flow channel) and outside of the fast flow channel in the $z$-direction, respectively. In Fig. 2, the one satellite

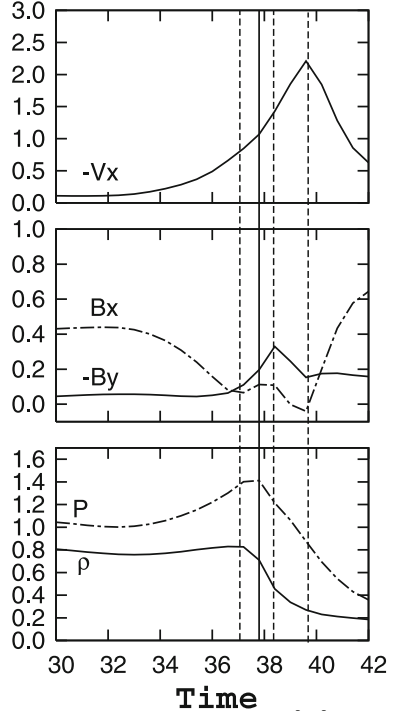

(a) $(\mathrm{x}=4.0, \mathrm{y}=0.4, \mathrm{z}=0.0)$
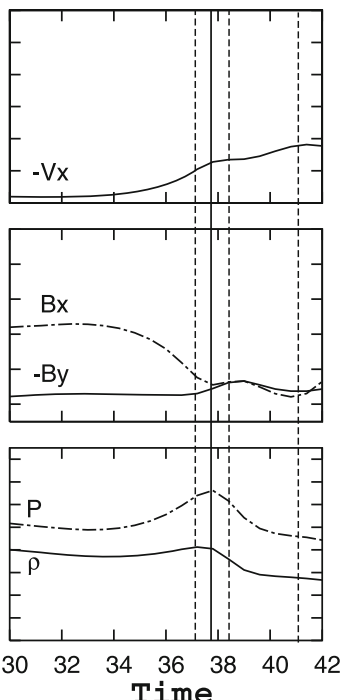

(b) $(\mathrm{x}=4.0, \mathrm{y}=0.4, \mathrm{z}=1.2)$
Fig. 3. Time variations of $-V_{x}, B_{x},-B_{y}, \rho$, and $P$ for the interval of $t=30$ to 42 at $X=4.0, Y=0.4, Z=0.0$ (a) and 1.2 (b).

was located in the $y=0$ plane in order to examine the profiles of the satellite observations at different $y$-positions. However, it is rare case that the actual satellites are located in the neutral sheet, that is, they observe no $B_{x}$ component. Therefore, these two virtual satellites are located in the $y=0.4$ plane. Figure 3 shows the temporal variations of the plasma flow velocity (top panel), magnetic field components (middle panel), plasma pressure and plasma density (bottom panel) detected by the virtual satellite located at $(4.0,0.4,0.0)$ (a) and $(4.0,0.4,1.2)(b)$. In the fast flow channel (a), the enhancement of earthward flow speed is large, but the satellite goes out the fast flow channel in the $y$-direction at $t \sim 40$. The plasma pressure and density decrease is significantly large. On the other hand, the enhancement of earthward flow speed observed outside the fast flow (b) is small, but the duration is very long because the satellite does not go out the fast flow channel in the $y$ direction easily due to the calm plasma sheet thinning. The decrease of plasma pressure and density observed outside the fast flow channel is not so large.

\section{Summary}

In this paper, the earthward fast flow events observed in the near-Earth plasma sheet were examined using three dimensional magnetohydrodynamics simulations on the basis of the spontaneous fast reconnection model. In particular, the properties of the variation of physical quantities in each observation point relative to the $\mathrm{X}$ reconnection line and the narrow channel of fast reconnection jet were investigated in three dimensions. The time variations of physical quantities observed by virtual satellites were quite different between the satellite positions in central plasma sheet and near the plasma sheet boundary layer. The satellite located in central plasma sheet does not go out the narrow channel of fast flow, so that the enhancement of earthward fast flow speed is large, and the decrease of plasma pressure and density is large. On the other hand, the satellite located near the 
plasma sheet boundary layer suddenly comes in the narrow channel and suddenly goes out, so that the enhancement of earthward fast flow speed is small and short, the decrease of earthward component of magnetic field is large in the course of fast flow, and the variations of plasma pressure and density are observed only in the course of fast flow. The time variations of physical quantities observed by virtual satellites were also quite different between the satellite positions in the sheet current direction. As mentioned in Introduction, the Alfvénic earthward fast jet flows in very narrow channel. In addition to this, relatively slow earthward jet (about $1 / 3$ of Alfvén speed) flows in wider region in the sheet current direction. In that region, plasma sheet thinning is also calm, so that the satellite located in that region does not go out that region easily. Therefore, the long duration enhancement of earthward flow is observed in that region. Let us interpret the positions of the satellites which observed the sample two events shown in Introduction on the basis of these results. The properties in Fig. 1(a) were long enhancement of earthward flow speed, small variation of earthward component of magnetic field, small increase of northward component of magnetic field, and small decrease of plasma density. These properties are same as that observed in the region near the central plasma sheet and outside of the narrow channel of the Alfvénic fast flow. On the other hand, the properties in Fig. 1(b) were short enhancement of earthward flow speed, very small variation of plasma density, and large variation of earthward component of magnetic field. These were clearly same as the results observed at the near plasma sheet boundary layer. In summary, we have demonstrated that the time variation of plasma quantities in the earthward fast flow event depends on the satellite position, in particular on the position relative to narrow channel of Alfvénic fast flow.

Acknowledgments. This work was supported by Grant-in-Aids for Creative Scientific Research 'The Basic Study of Space Weather Prediction' (17GS0208, Head Investigator: K. Shibata) from the Ministry of Education in Japan, Mitsubishi Foundation, RISH of Kyoto University, and Solar Terrestrial Environment Laboratory of Nagoya University. The computer program was tested and run at the Computational Centers of Nagoya and Kyoto University. Geotail magnetic field and plasma data were provided by T. Nagai and T. Mukai through DARTS at Institute of Space and Astronautical Science, JAXA in Japan.

\section{References}

Angelopoulos, V., W. Baumjohann, C. F. Kennel, F. V. Coroniti, M. G. Kivelson, R. Pellat, R. J. Walker, H. Luhr, and G. Pashmann, Bursty bulk flows in the inner central plasma sheet, J. Geophys. Res., 97, 40274039, 1992.

Kondoh, K. and M. Ugai, Study of three dimensional structure of the fast convection flow in the plasma sheet by MHD simulations on the basis of spontaneous fast reconnection model, Adv. Space Res., 39, 1378-1381, 2007.

Petschek, H. E., Magnetic field annihilation, Magnetic Field Annihilation, NASA Spec. Publ. SP-50, 425-439, 1964.

Sergeev, V. A., J.-A. Sauvaud, D. Popescu, R. A. Kovrazhkin, K. Liou, P. T. Newell, M. Brittnacher, G. Parks, R. Nakamura, T. Mukai, and G. D. Reeves, Multiple-spacecraft observation of a narrow transient plasma jet in the Earth's plasma sheet, Geophys. Res. Lett., 27, 851-854, 2000.

Shiokawa, K. et al., Braking of high-speed flows in the near-Earth tail, Geophys. Res. Lett., 24, 1179-1182, 1997.

Ugai, M., Self-consistent development of fast magnetic reconnection with anomalous plasma resistivity, Plasma Phys. Controlled Fusion, 26, $1,549-1,563,1984$.

Ugai, M., Global dynamics and rapid collapse of an isolated current-sheet system enclosed by free boundaries, Phys. Fluids, 29, 3,659-3,667, 1986

Ugai, M. and K. Kondoh, Computer studies on the spontaneous fast reconnection evolution in various physical situations, Phys. Plasmas, $\mathbf{8}$, $1,545-1,552,2001$.

Ugai, M., K. Kondoh, and T. Shimizu, Computer simulations on threedimensional magnetic loop dynamics by the spontaneous fast reconnection model, Phys. Plasmas, 10, 357-363, 2003.

Ugai, M., K. Kondoh, and T. Shimizu, Computer studies on the threedimensional spontaneous fast reconnection model as a nonlinear instability, Phys. Plasmas, 11, 1,416-1,423, 2004.

Ugai, M., K. Kondoh, and T. Shimizu, Spontaneous fast reconnection model in three dimensions, Phys. Plasmas, 12, 042903, 2005.

K. Kondoh (e-mail: kondo@cs.ehime-u.ac.jp), M. Ugai, and T. Shimizu 